\title{
Forward
}

\section{Why a special issue of JIDC on enteric fever?}

\author{
John Wain, on behalf of the editorial board of JIDC
}

J Infect Developing Countries 2008; 2(4): 249.

Received 20 August 2008 - Accepted 20 August 2008

Copyright $(2008$ Wain. This is an open access article distributed under the Creative Commons Attribution License, which permits unrestricted use, distribution, and reproduction in any medium, provided the original work is properly cited.

The Journal of Infection in Developing Countries (JIDC) was started as a vehicle to promote the work of developing country scientists and to enable research in the less affluent regions of the world. This issue of JIDC is focused on enteric fever, a disease seen predominantly in developing countries. The idea came out of discussions through the open forum, the Salmonella network, and at editorial meetings. The hope is that we have put together a collection of state-of-the-art reviews on relevant topics; that is, topics that are relevant to basic research and public health, written by scientists currently working at the top of their field. This collection is complemented by regional reviews which give a view from endemic regions of the world and, particularly for sub-Saharan Africa and China, provide data on the importance of enteric fever which was previously unavailable in the West. Finally, we have also received many original research articles which provide the most recent data on many aspects of enteric fever.
Our journal, JIDC, is growing and there is a genuine commitment to address our aim of "bridging the gap between developed and developing country scientists." I have been overwhelmed by the enthusiastic response from both developing and developed country scientists for this issue and would like to thank all of those who submitted manuscripts and acted as reviewers and associate editors. I apologize to those authors whose papers did not make the deadline for this issue, but we will try to publish the manuscripts on enteric fever which are still being processed as soon as possible. Please continue to submit articles, on any relevant topic, but also on enteric fever, as we are hoping to collate all of the articles in this special issue of JIDC on enteric fever into a single publication for release at the international typhoid meeting in Kenya in January.

Corresponding Author: John Wain, Wellcome Trust Sanger Institute, Hinxton Cambs. CB10 1SA. Email jw5@sanger.ac.uk

Conflict of interest: No conflict of interest is declared. 